\title{
BCH Two-step Auxiliary Variable Integration with MplusAutomation
}

Adding covariates and distal outcome variables to mixture models

\author{
Adam Garber
}

$9 / 8 / 2021$

This R tutorial automates the $\mathrm{BCH}$ two-step axiliary variable procedure (Bolk, Croon, Hagenaars, 2004) using the MplusAutomation package (Hallquist \& Wiley, 2018) to estimate models and extract relevant parameters. To learn more about auxiliary variable integration methods and why multi-step methods are necessary for producing un-biased estimates see Asparouhov \& Muthén (2014). The name of this mehtod, BCH, stands for Bolck, Croon, \& Hagenaars, the authors who developed this method (Bolk, Croon, Hagenaars, 2004).

Data source \& applied example. This tutorial utilizes the public-use data repository named the Longitudinal Survey of American Youth (LSAY; Miller et al., 1992). The applied example used in this tutorial is based off the example presented in the seminal chapter on mixture modeling by Katherine Masyn (2013). This tutorial contains the 9 math indicators from this original study as well as two auxiliary variables. See the following book chapter for details about the applied example:

Masyn, K. E. (2013). Latent class analysis and finite mixture modeling. In T. D. Little (Ed.), The Oxford Handbook of Quantitative Methods: Vol. 2. Statistical analysis (pp. 551-611). New York, NY: Oxford University Press. http://dx.doi.org/10.1093/oxfordhb/9780199934898.013.0025

If using this tutorial to automate the 3-step approach it would be greatly appreciated if you cite this resource:

Garber, A. C. (2021). BCH Two-step Auxiliary Variable Integration Using MplusAutomation. Retrieved from https://psyarxiv.com/wmfcj

Associated Github repository:

https://github.com/garberadamc/BCH-Method 
Load packages

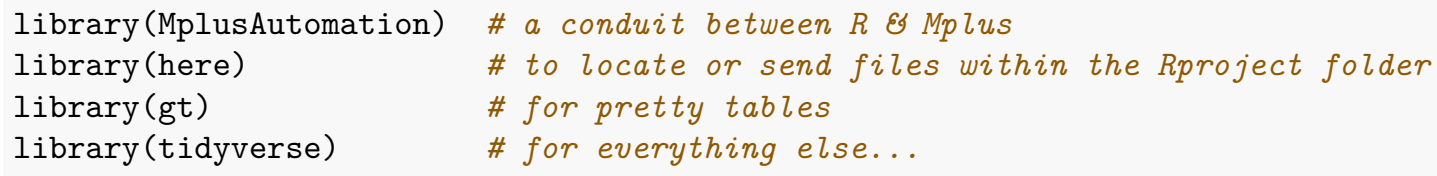

Read in LSAY data lsay_data <- read_csv(here("data", "lsay_subset.csv")) \% $\%$
mutate $(f e m a l e=$ gender - 1)

\section{LCA Indicators \& Auxiliary Variables: Math Attitudes Example ${ }^{1}$}

\begin{tabular}{ll}
\hline Name & Variable Description \\
\hline 7 th Grade Student Math Attitudes & \\
\hline enjoy & I enjoy math. \\
good & I am good at math. \\
undrstnd & I ussually understand what we are doing in math. \\
nervous & Doing math often makes me nervous or upset \\
scared & I often get scared when I open my math book and see a page of problems. \\
useful & Math is useful in everyday problems. \\
logical & Math helps a person think logically. \\
job & It is important to know math to get a good job. \\
adult & I will use math in many ways as an adult. \\
\hline Auxiliary Variables & \\
\hline female & \\
math_irt & Self-reported student gender $(0=$ Male, $1=$ Female). \\
\hline
\end{tabular}

${ }^{1}$ Note. The example presented replicates the model found originally in Masyn (2013). All data is from the public-use dataset, the Longitudinal Survey of American Youth (LSAY; Miller et al., 1992) 


\section{"Manual BCH Two-step" Auxiliary Variable Integration Method}

Step 1 - Estimate the unconditional model with all covariate \& distal outcome variables mentioned in the auxiliary statement.

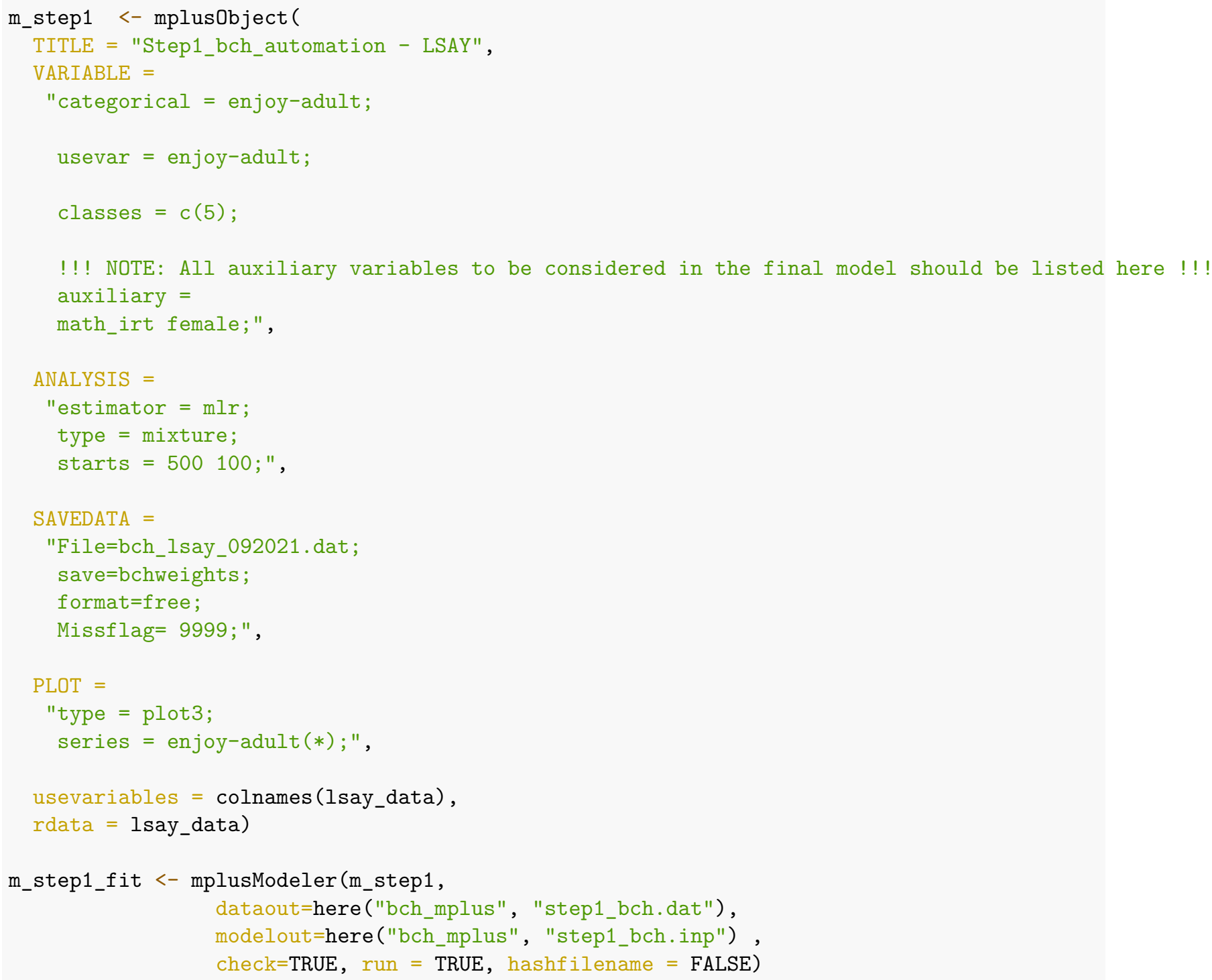

Extract saved data from the step 1 unconditional model. 
Extract saved data from the step 1 model mplusObject named "m_step1_fit"

savedata <- as.data.frame(m_step1_fit [["results"]]

$[$ ["savedata"] ])

Rename the column in savedata for "C" and change to "N"

colnames $($ savedata $)[$ colnames $($ savedata $)==" C "]<-~ " N "$ 


\section{Step 2 - Estimate the model with auxiliary variables using BCH weights}

Example demonstrated is a moderation model with covariate \& distal outcome.

\section{Specification details:}

- This example contains one distal outcome variable (math_irt) and one binary covariate (female).

- Under each class-specific statement (e.g., \%C\#1\%) the distal outcome is mentioned to estimate the intercept mean (in square brackets) \& variance parameters.

- Moderation is specified by mentioning the "outcome ON covariate;" syntax under each of the classspecific statements.

- Note that the binary covariate is centered so that reported distal means (intercepts) are estimated at the weighted average of female.

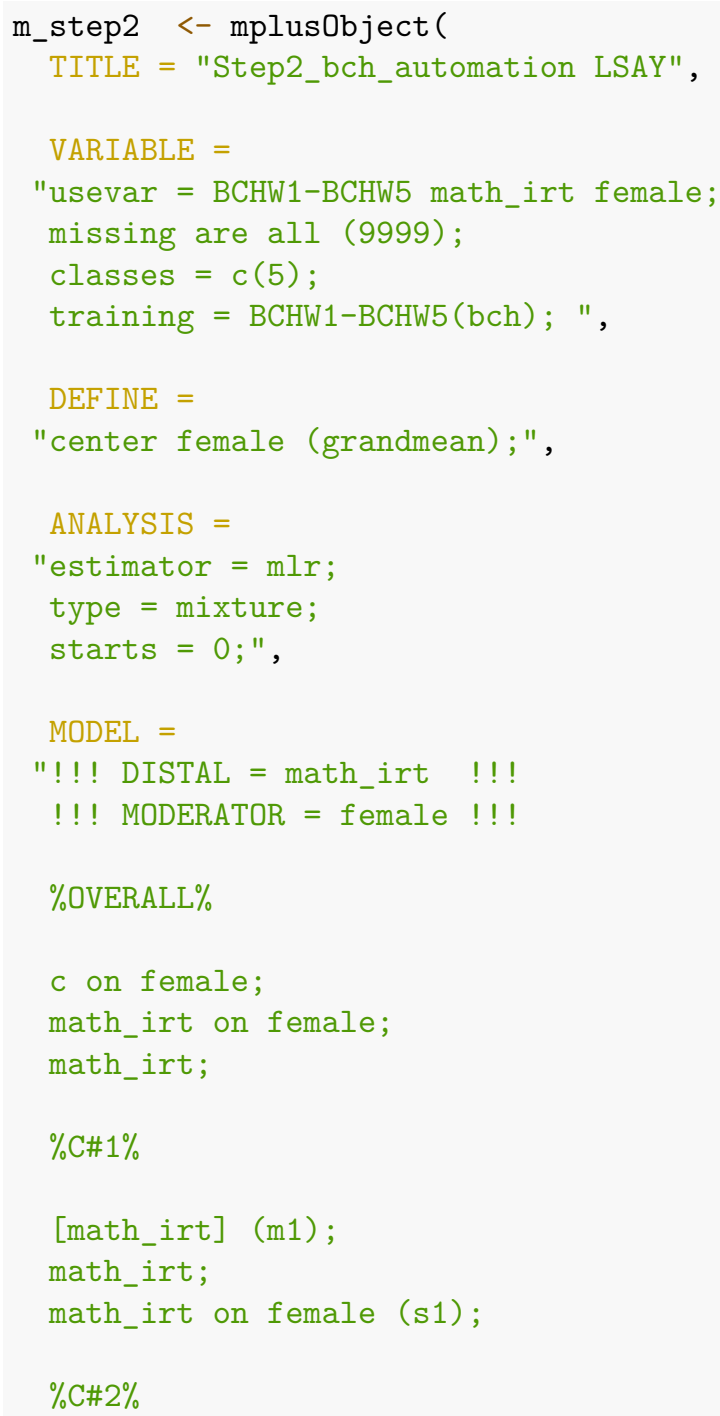




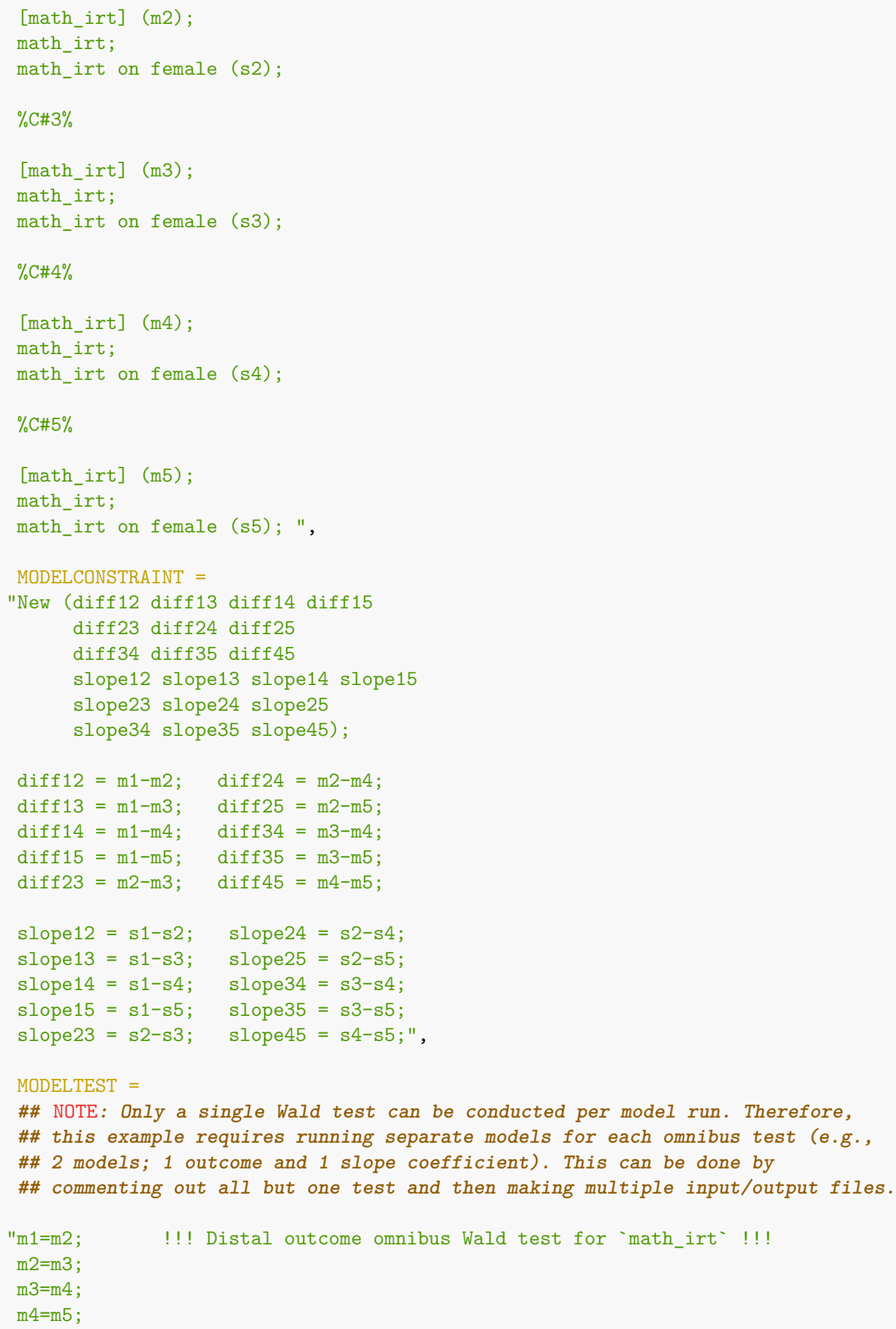




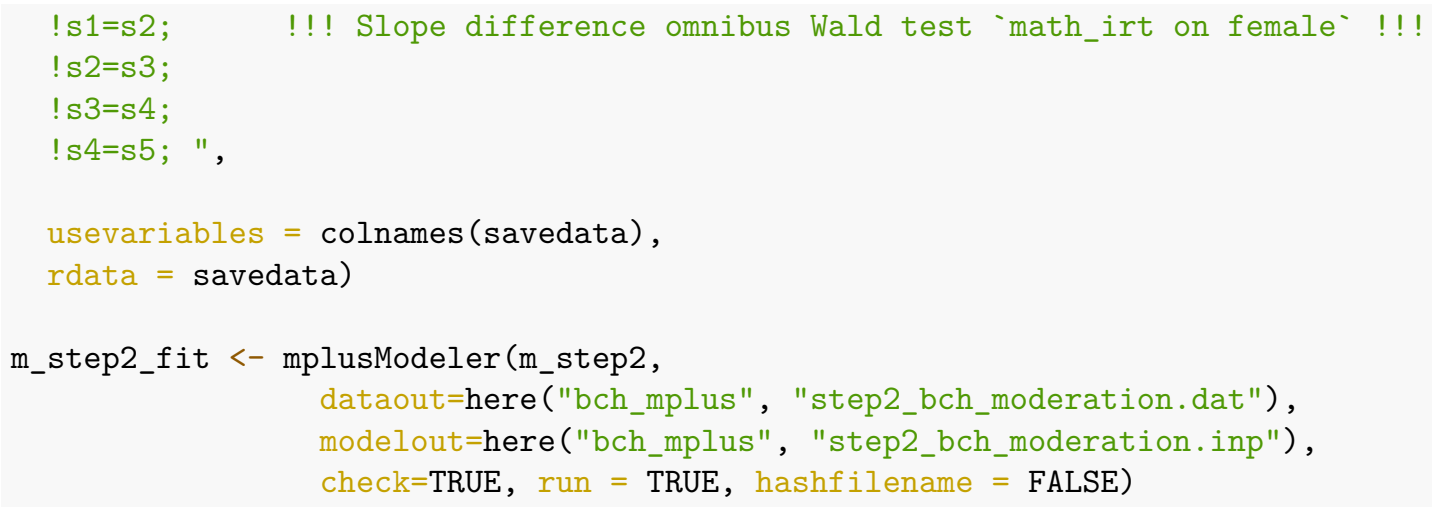

\section{References}

Bolck, A., Croon, M., \& Hagenaars, J. (2004). Estimating latent structure models with categorical variables: One-step versus three-step estimators. Political analysis, 12(1), 3-27.

Hallquist, Michael N., and Joshua F. Wiley. 2018. "MplusAutomation: An R Package for Facilitating Large-Scale Latent Variable Analyses in Mplus." Structural Equation Modeling, 1-18. https://doi.org/10. 1080/10705511.2017.1402334.

Nylund, K. L., Asparouhov, T., \& Muthén, B. O. (2007). Deciding on the number of classes in latent class analysis and growth mixture modeling: A Monte Carlo simulation study. Structural equation modeling: A multidisciplinary Journal, 14(4), 535-569.

Masyn, K. E. (2013). Latent class analysis and finite mixture modeling. In T. D. Little (Ed.), The Oxford Handbook of Quantitative Methods: Vol. 2. Statis- tical analysis (pp. 551-611). New York, NY: Oxford University Press. http://dx.doi.org/10.1093/oxfordhb/9780199934898.013.0025

R Core Team. 2019.R: A Language and Environment for Statistical Computing. Vienna, Austria: R Foundation for Statistical Computing. https://www.R-project.org/.

Wickham H et al., (2019). "Welcome to the tidyverse." Journal of Open Source Software, 4(43), 1686. doi: 10.21105 /joss. 01686 .

For more examples using MplusAutomation:

https://garberadamc.github.io/project-site/ 